\title{
All-optical calculus based on dynamic Brillouin grating reflectors in optical fibers
}

\author{
Nikolay Primerov, Sanghoon Chin, Luc Thévenaz \\ Ecole Polytechnique Fédérale de Lausanne, STI-GR-SCI-LT Station 11, CH-1015 Lausanne, Switzerland \\ Leonora Ursini, Marco Santagiustina \\ CNIT-Department of Information Engineering, University of Padova, via Gradenigo 6b, 35131, Padova, Italy \\ nikolay.primerov@epfl.ch
}

\begin{abstract}
We experimentally demonstrate that all-optical signal calculus can be realized based on dynamic Brillouin gratings in fibers. Temporal integration and first-order differentiation were performed for optical pulse with various waveforms.

OCIS codes: (060.4370) Nonlinear optics, fibers; (290.5900) Scattering, stimulated Brillouin; (070.6020) Signal processing
\end{abstract}

Development of all-optical temporal integrator and differentiator in photonic signal processing circuit has received a great attention in the optical community, since they are fundamental functions required for all-optical computing systems and networks. In this paper, we experimentally demonstrate a new type of all-optical differentiator and integrator based on dynamic Brillouin gratings (DBGs) in fibers. The proposed operators are conceptually similar to previously developed systems using uniform long period gratings and weak fiber Bragg gratings [1,2], since DBGs can considered as a weak fiber Bragg grating [3]. However, this type of function has a crucial advantage: the grating length can be flexibly tuned so as to properly adopt a wide-range signal bandwidth. Moreover, arbitrarily long gratings are possible to integrate a long sequence of signal pulses.

\section{- DBG-based optical signal integration}

Optical signal integration in our system is actually based on two different physical processes. First, an acoustic wave was uniformly created in a $42 \mathrm{~cm}$-long polarization maintaining fibers (PMFs), resulting from the stimulated Brillouin scattering (SBS) interaction between two continuous wave pumps counter-propagating through the PMF along one principle polarization axis. Then the acoustic wave acts as a grating reflector with weak reflectivity. This grating can reflect a light at distinct optical frequency to the pump frequencies, but orthogonally polarized with respect to the pumps. This optically generated grating is denominated DBG. Second, a signal pulse spectrally centered at DBG was launched into the PMF, and then the distributed pulse reflection corresponds to the temporal integration of the input signal. Time integrations of two different signal waveforms a 800 ps single pulse and 300 ps double pulses, spaced by 900 ps - were measured, as shown in Figure 1(a) and 1(b). The experimental results show a good agreement with numerically calculated integration of the waveforms.

\section{- DBG-based optical signal differentiation}

Time differentiation is realized using a slightly modified configuration of the coherent light storage technique [4]. A $7 \mathrm{~ns}$ optical pulse was launched into a $20 \mathrm{~m}$-long PMF and its waveform was stored along the fiber using a 800 ps writing pulse, in the form of an acoustic wave as a result of the SBS interaction. Double 800 ps pulses with a time interval of $800 \mathrm{ps}$ are used as a reading pulse, but the two pulses are $\pi$ phase shifted in amplitude. Each pulse is then reflected by the DBG, resulting in two identical signal waveforms with a time delay of $800 \mathrm{ps,}$ but with opposite sign. The delayed destructive interference between the two waveforms eventually represents the first-order derivative of the signal waveform, as shown in Figure 1(c).
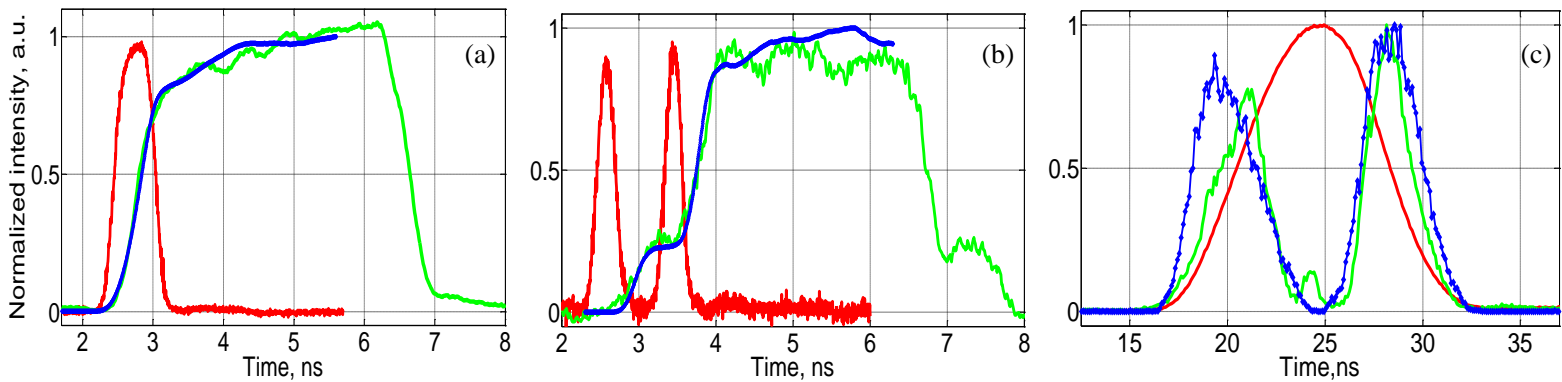

Fig. 1. (a) and (b): Measured (green) and calculated (blue) time traces of the transformed input signal (red) for temporal time integration $(\mathrm{a}, \mathrm{b})$ and derivative (c). The discrepancy between measure and calculation in (c) is certainly due to a step phase shift smaller than $\pi$.

\section{References}

[1] R. Slavík, Y. Park, M. Kulishov, R. Morandotti and J.Azaña, "Ultrafast all-optical differentiators,” Opt. Express 14, 10699-10707 (2006).

[2] Y. Park, T-J. Ahn, Y. Dai, J. Yao and J. Azaña, "All-optical temporal integraton of ultrafast pulse waveforms," Opt. Express 16, 1781717825 (2008).

[3] K. Y. Song and H. J. Yoon, "Observation of narrowband intrinsic spectra of Brillouin dynamic gratings," Opt. Lett. 35, 2958-2960 (2010).

[4] Z. Zhu, D. J. Gauthier, R. W. Boyd, "Stored light in an optical fiber via stimulated Brillouin scattering," Science 318, 1748-1750 (2007). 